\title{
Complex-Temperature Partition Function Zeros of the Potts Model on the Honeycomb and Kagomé Lattices
}

\author{
Heiko Feldmann*, Robert Shrock ${ }^{\dagger}$, Shan-Ho Tsai ${ }^{\ddagger}$ \\ Institute for Theoretical Physics \\ State University of New York \\ Stony Brook, N. Y. 11794-3840
}

\begin{abstract}
We calculate complex-temperature $(\mathrm{CT})$ zeros of the partition function for the $q$-state Potts model on the honeycomb and kagomé lattices for several values of $q$. These give information on the CT phase diagrams. A comparison of results obtained for different boundary conditions and a discussion of some CT singularities are given. Among other results, our findings show that the Potts antiferromagnet with $q=4$ and $q=5$ on the kagomé lattice has no phase transition at either finite or zero temperature.

05.20.-y, 64.60.C, 75.10.H
\end{abstract}

*email: feldmann@insti.physics.sunysb.edu

†email: shrock@insti.physics.sunysb.edu

‡email: tsai@insti.physics.sunysb.edu 


\section{INTRODUCTION}

The 2D $q$-state Potts models [1,2] for various $q$ have been of interest as examples of different universality classes for phase transitions and, for $q=3,4$, as models for the adsorption of gases on certain substrates. The $q=2$ Ising special case has long served as a simple exactly solvable model of cooperative phenomena. However, for $q \geq 3$, the free energy of the Potts model has never been calculated in closed form for arbitrary temperature. It is thus worthwhile to obtain further information about the properties of the Potts model, and we shall do this in the present paper via calculations of complex-temperature (CT) zeros of the partition function of the Potts model for the honeycomb and kagomé lattices. One of the motivations for this work is the recent calculation and analysis of long low-temperature series for the $q$-state Potts model on these lattices by Jensen, Guttmann, and Enting [3]. Our results enable one to relate the CT singularities in thermodynamic quantities found in Ref. [3] to positions on the CT phase boundaries of the respective models.

The study of statistical mechanical models with magnetic field [4], temperature [5]- [8], or both [9] generalized from real to complex values has yielded interesting insights into the properties of these models. For a discrete spin model at temperature $T$ and in an external magnetic field $H$, the partition function $Z$ is, up to a prefactor, a polynomial in the Boltzmann weights $z(K)$ and $\mu(h)$ containing dependence on $K=\beta J$ and $h=\beta H$, where $\beta=1 /\left(k_{B} T\right)$, and $J$ is the spin-spin coupling. It is of interest to study the zeros of $Z$ (i) in the complex $\mu$ plane for physical $T$ [4]; (ii) in the complex $z$ plane for physical (vanishing or nonvanishing) $H$ [5]; and (iii) on the $\mathbb{C}^{2}$ manifold $(\mu, z)$ when both $K$ and $h$ are complex [9]. Here we shall concentrate on case (ii), i.e., Fisher zeros. In the thermodynamic limit, via a coalescence of zeros, there forms a continuous locus $\mathcal{B}$ of points where the free energy is nonanalytic. This locus serves as the union of boundaries (whence the symbol $\mathcal{B})$ of the various complex-temperature phases. Thus, calculations of complex-temperature partition function zeros on sufficiently large finite lattices yield useful information on the CT phase diagram in the thermodynamic limit. (Hereafter, to avoid repetition, we shall simply refer to zeros of the partition function, it being understood that these are complextemperature zeros.) In making inferences from such finite-lattice calculations about $\mathcal{B}$ in the thermodynamic limit it is important to vary both the lattice size and the type of boundary conditions to have an accurate idea of the sensitivity of the locations of the zeros to these choices. Some of the earliest work on CT properties of spin models dealt with these zeros [5, 6]. Another major reason for early interest in these properties of spin models was the fact that

unphysical, CT singularities complicated the analysis of low-temperature series expansions to get information about the location and exponents of the physical phase transition [8]. A third 
reason for interest in these properties is the fact that, as additional sources of information about thermodynamic functions, they can expedite progress toward exact solutions. Aside from well-understood exceptions [10], CT singularities of thermodynamic functions occur on the continuous locus of points $\mathcal{B}$ where the free energy is nonanalytic. Hence, when investigating these singularities, it is useful to do so in conjunction with a calculation of the zeros of the partition function to infer the approximate location of the phase boundary $\mathcal{B}$ separating various CT phases [12]. Interestingly, some of these singularities can be related directly to physical singularities: by using duality, one can show an exact equivalence of the free energy of the $q$-state Potts antiferromagnet on a lattice $\Lambda$ for the full temperature interval $0 \leq T \leq \infty$ and the free energy of the $q$-state Potts model on the dual lattice for a semi-infinite interval of complex temperatures [13]. This implies the existence of two quite different types of CT singularities: the generic kind, which does not obey universality or various scaling relations 14,15, and a special kind which does obey such properties and encodes information of direct physical relevance. Although we consider the honeycomb and kagomé lattices here, we mention that previous calculations of zeros of the partition function for the Potts model with $q \geq 3$ have been done on the triangular and square lattices [16 19].

\section{MODEL}

The (isotropic, nearest-neighbor) $q$-state Potts model on a lattice $\Lambda$ is defined by the partition function

$$
Z=\sum_{\left\{\sigma_{n}\right\}} e^{-\beta \mathcal{H}}
$$

with the Hamiltonian

$$
\mathcal{H}=-J \sum_{\left\langle n n^{\prime}\right\rangle} \delta_{\sigma_{n} \sigma_{n^{\prime}}}-H \sum_{n} \delta_{1 \sigma_{n}}
$$

where $\sigma_{n}=1, \ldots, q$ are $\mathbb{Z}_{q}$-valued variables on each site $n \in \Lambda, \beta=\left(k_{B} T\right)^{-1}$, and $\left\langle n n^{\prime}\right\rangle$ denotes pairs of nearest-neighbor sites. The symmetry group of the Potts Hamiltonian is the symmetric group on $q$ objects, $S_{q}$. We use the notation introduced above, $K=\beta J, h=\beta H$, and

$$
\begin{gathered}
a=z^{-1}=e^{K} \\
x=\frac{e^{K}-1}{\sqrt{q}}
\end{gathered}
$$


The (reduced) free energy per site is denoted as $f=-\beta F=\lim _{N_{s} \rightarrow \infty} N_{s}^{-1} \ln Z$, where $N_{s}$ denotes the number of sites in the lattice. There are actually $q$ types of external fields which one may define, favoring the respective values $\sigma_{n}=1, . ., q$; it suffices for our purposes to include only one. The order parameter (magnetization) is defined to be $m=(q M-1) /(q-1)$. where $M=\langle\sigma\rangle=\lim _{h \rightarrow 0} \partial f / \partial h$. With this definition, $m=0$ in the symmetric, disordered phase, and $m=1$ in the limit of saturated ferromagnetic (FM) long-range order. We consider the zero-field model, $H=0$. For $J>0$ and the dimensionality of interest here, $d=2$, the $q$-state Potts model has a phase transition from the symmetric, high-temperature paramagnetic (PM) phase to a low-temperature phase involving spontaneous breaking of the $S_{q}$ symmetry and onset of ferromagnetic (FM) long-range order. This transition is continuous for $2 \leq q \leq 4$ and first order for $q \geq 5$. The critical exponents and universality classes of the cases where the model has second-order transitions are well understood [2,20]. The $q$-state Potts model has the property of duality [1,2, 21,22], which relates the partition function on a lattice $\Lambda$ with temperature parameter $a$ to the partition function on the dual lattice with temperature parameter

$$
a_{d} \equiv \mathcal{D}(a)=\frac{a+q-1}{a-1}, \quad \text { i.e. } \quad x_{d}=\frac{1}{x}
$$

Other exact results include formulas for the PM-FM transition temperature on the square, triangular, and honeycomb lattices [1, 2, 22], and calculations of the free energy at the phase transition temperature, and of the related latent heat for $q \geq 5$ [23]. No formula is known for the PM-FM transition on the kagomé lattice, although there have been a number of conjectures; for a recent discussion, see Ref. [3]. The case $J<0$, i.e., the Potts antiferromagnet (AF) has also been of interest because of its connection with graph colorings and the fact that, for certain lattices and values of $q$, it exhibits nonzero ground state entropy 24,25]; for a recent discussion, see [26,27] and references therein. Depending on the type of lattice and the value of $q$, the model can also have a phase with AFM long-range order. For $q \geq 3$ on the honeycomb lattice there is no AFM phase [28,29. For any lattice $\Lambda$, the partition function can be expressed in a form involving a sum of powers of $q$ which allows a generalization from positive integer $q$ to real (or, indeed, complex) $q$, and we shall use the generalization to real $q$ at certain places below. Reviews of the model include Refs. [2, 17].

On a finite lattice, the $q$-state Potts model partition function $Z$ is a polynomial in the Boltzmann weight $a$. We calculate this polynomial by transfer matrix methods. This is a challenging numerical problem for large lattices, since the degree of the polynomial is equal to the number of bonds, $N_{b}=(\Delta / 2) N_{s}$, where $\Delta$ is the coordination number, and there is a very large range in the sizes of the coefficients, from $q$ for the highest-degree term $a^{N_{b}}$ to exponentially large values for intermediate terms. The latter property is obvious from the 
fact that for $K=0$, i.e., $a=1$, the sum of the coefficients in $Z$ is $q^{N_{s}}$. From this, we then compute the zeros. A general property of the CT phase boundary for any lattice and $q$ value is invariance under complex-conjugation: $\mathcal{B} \rightarrow \mathcal{B}$ as $a \rightarrow a^{*}$.

In addition to the locations of the curves comprising the $\mathrm{CT}$ phase boundary $\mathcal{B}$ inferred in the thermodynamic limit from the zeros calculated on finite lattices, one can extract further information. As one approaches the thermodynamic limit, so that one can define a density of zeros, this density normally behaves near a singular point $a_{s}$ as [5,7]

$$
g(s) \sim s^{1-\alpha}, \quad \text { as } \quad s \rightarrow 0
$$

where $s$ denotes the arclength along $\mathcal{B}$ away from $a_{s}$ (so that $s=\left|a-a_{s}\right|$ as $s \rightarrow 0$ ) and where the singularity in the free energy at $a_{s}$ is $f_{\text {sing }} \sim\left|a-a_{s}\right|^{2-\alpha}$ [30]. If the partition function has a zero at some point $z_{0}$ with a multiplicity proportional to the number of lattice

sites, $N_{s}$, then this formula, eq. (2.6), is modified by the addition of a term proportional to a delta function $\delta(s)$. In Ref. [11] it was proved (as Theorem 6) that for the Ising model on a lattice with odd coordination number, this happens at $z=-1$. In particular, this occurs for the Ising model on the honeycomb lattice (see further below).

\section{PARTITION FUNCTION ZEROS ON THE HONEYCOMB LATTICE}

\section{A. Comparison with Exact $\mathcal{B}$ for Ising Case}

In order to study the effects of the finite lattice size and of different boundary conditions, as well as checking the computer programs used, it is valuable to calculate the zeros for the $q=2$ Ising case where the resulting locus of zeros can be compared with the exactly known CT phase boundary $\mathcal{B}$. As noted above, these zeros, like the others to be presented further below, are calculated by a transfer matrix method. From the known expression for the free energy, this boundary was determined in Ref. [31]; it is the locus of solutions to the equation

$$
1-2 a+6 a^{2}-2 a^{3}+a^{4}-2 a(1-a)^{2} p=0
$$

where $-3 / 2 \leq p \leq 3$ [32. This locus is shown in Figs. 2 and 3. Because $q=2$ and the honeycomb lattice is bipartite, the $\mathrm{CT}$ phase boundary $\mathcal{B}$ and also the set of zeros are invariant under the inversion map [33]. The CT boundary consists of the union of two parts. The first is an arc of the unit circle extending from $\theta=\arg (a)=\pi / 3$ around through $a=-1$ to $\theta=-\pi / 3$, while the second is a lima bean-shaped curve that crosses the positive real axis at the PM-FM critical point, $a_{P M-F M, q=2}=2+\sqrt{3}=3.732 \ldots$ and at the PM-AFM critical 
point, $a_{P M-A F M, q=2}=a_{P M-F M, q=2}^{-1}=2-\sqrt{3}=0.267949 \ldots$. These two parts intersect each other at multiple points at $\pm i$; these multiple points are singular points of $\mathcal{B}$ in the sense of algebraic geometry [35]. The phase surrounding the origin in the $a$ plane is the AFM phase; the one surrounding the infinite-temperature point $a=1$ is the PM phase, and the one extending outside $\mathcal{B}$ to complex infinity in all directions is the FM phase [12].

Before we start to present our results, we have to introduce our notation for the sizes and orientations of the lattices. We recall first that the (infinite) honeycomb lattice is a homopolygonal member, and the kagomé lattice, a heteropolygonal, member of the class of Archimedean lattices, i.e., regular tiling of the plane by one or more types of regular polygons such that every vertex is equivalent to every other vertex [36]. An Archimedean lattice is thus uniquely defined by the ordered sequence of polygons that one traverses in making a circuit of any vertex. In standard mathematical notation [36], such a lattice is denoted $\Lambda=\left(\prod_{i} p_{i}^{a_{i}}\right)$ where $p_{i}$ refers to the type of polygon and $a_{i}$ denotes the number of times that it appears consecutively in the product. In this notation, the kagomé lattice is denoted $(3 \cdot 6 \cdot 3 \cdot 6)$. The homopolygonal subset of Archimedean lattices (consisting of tilings with only one type of regular polygon) is closed under duality, but the heteropolygonal Archimedean lattices (consisting of regular tilings using more than one type of polygon) have duals that are not Archimedean lattices. In particular, the dual of the kagomé lattice, called the diced lattice, is not Archimedean. This mathematical background will be useful below when we give results for the diced lattice. To indicate the size of a given lattice for both the honeycomb and kagomé cases, we count the number of hexagons. As an illustration, the sizes of the honeycomb and kagomé lattices in Fig. 1 are $4 \times 3$ and $3 \times 4$ hexagons, respectively. The number of sites in a lattice is also dependent on the boundary conditions: with periodic boundary conditions in the horizontal direction for example, the sites on the left and right are identified, while with free boundary conditions they are counted independently from each other. As is evident from Fig. 1, a honeycomb lattice of size, in our notation, $N_{x} \times N_{y}$, is maximally square-like if one takes $N_{x}$ slightly larger than $N_{y}$.
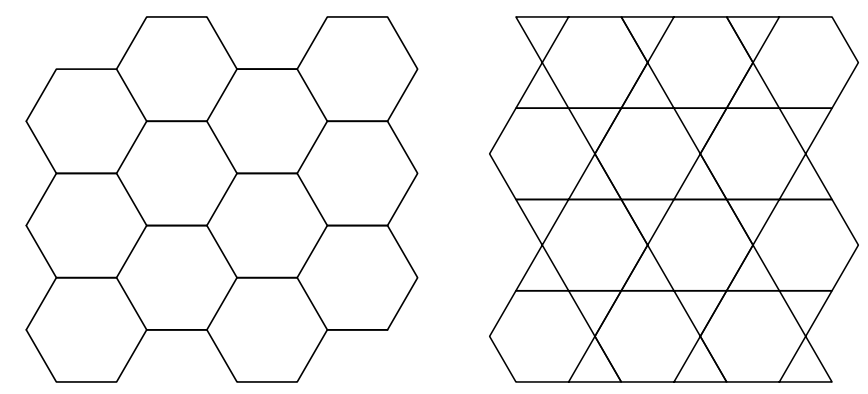

FIG. 1. Honeycomb and kagomé lattices to illustrate our conventions for indicating sizes. 
Since we use duality at many points in this work, we chose lattices that have natural dual lattices. This excludes lattices that are periodic in both directions, for the following reason: duality relies on the fact that every closed polygon divides the lattice into at least two regions. However, a lattice with periodic boundary conditions in both directions, and hence with toroidal geometry, has the property that there exist closed contours that do not divide the surface into two disjunct regions. Since boundary effects are, in general, best suppressed if one uses periodic boundary conditions in as many directions as possible, we use boundary conditions that are periodic in one direction and free in the other, i.e., cylindrical boundary conditions. Our notation for the boundary conditions (BC's) is (fbc,pbc) for free and periodic BC's in the horizontal $(x)$ and vertical $(y)$ directions, respectively (see Fig. 1), and $(\mathrm{pbc}, \mathrm{fbc})$ for periodic and free BC's in the $x$ and $y$ directions. This notation makes explicit the direction in which the cylindrical boundary conditions are periodic.

Complex-temperature partition function zeros for the $q=2$ Ising case of the Potts model on the honeycomb lattice are shown in Fig. 2 for both (fbc,pbc) and (pbc, fbc). The gray curves are the exactly known $\mathrm{CT}$ phase boundary $\mathcal{B}$. We remark on several features.

- The partition function has a multiple zero at $z=-1$ with multiplicity $\propto N_{s}$ for large $N_{s}$ This follows from Theorem 6 of Ref. [1] and corresponds to the term $\propto\left(1-z^{2}\right)^{-2}$ in the expression for the specific heat $C$ in the FM phase given as eq. (3.12) in Ref. [31]; the apparent additional singularity at the infinite-temperature point $z=1$ is not relevant since the formula does not apply in that region.

- The zeros lie very close to the arcs protruding into the PM phase.

- There seems to be some repulsion of the zeros from the multiple points at $a= \pm i$ (similar to what was seen in Ref. [9]).

- For the (fbc, pbc) case, the zeros on the unit circle show no radial deviation.

- In general, the zeros calculated with the choice (fbc, pbc) lie closer to the exact boundary curves $\mathcal{B}$ than those calculated with (pbc, fbc).

- The zeros lie on the outer side of the boundary between the PM and FM phase. This can be understood as a consequence of the fact that with either the (fbc,pbc) or (pbc,fbc) boundary conditions, the sites on the free boundary have a coordination number of two rather than the usual $\Delta=3$ for sites on an infinite honeycomb lattice. Hence, ordering effect of the spin-spin interactions is commensurately reduced, thereby reducing the finite-lattice manifestations of the ordered, FM phase, i.e. shifting the PM-FM boundary outwards. 

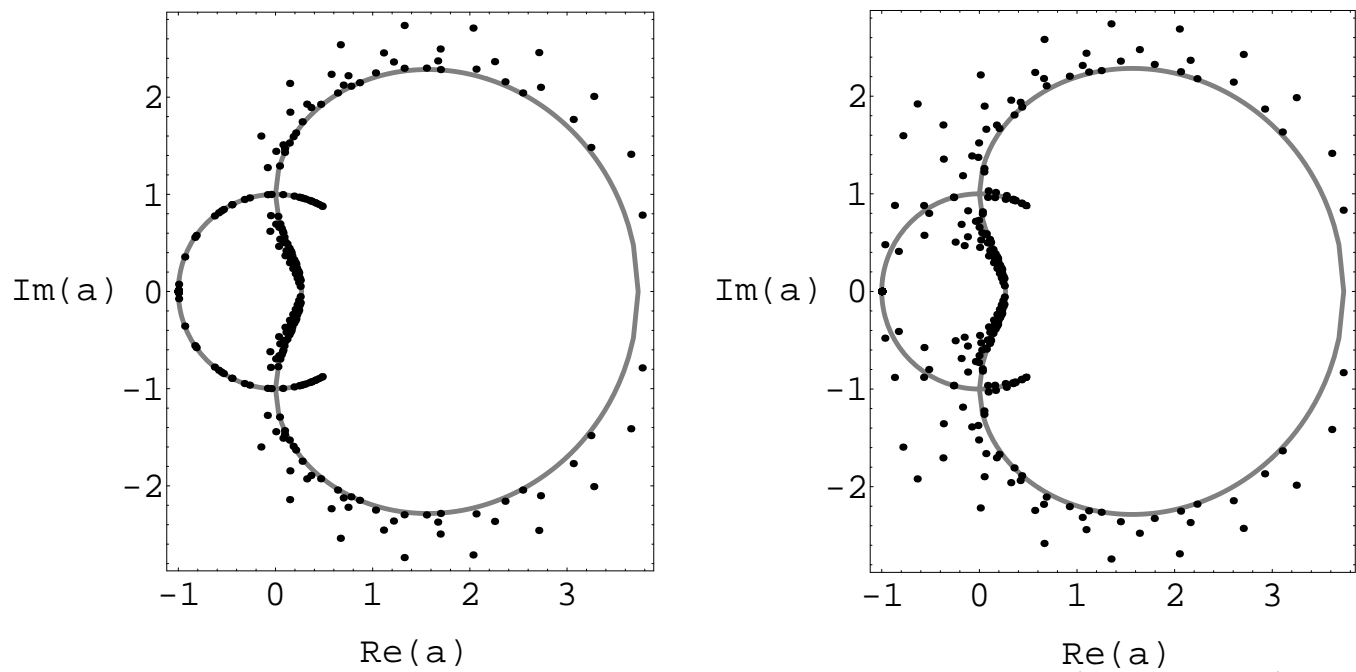

FIG. 2. Partition function zeros in the complex $a$ plane for the Ising ( $q=2$ Potts) model on a honeycomb lattice. (a) left: size $9 \times 12$ hexagons and (fbc, pbc); (b) right: $10 \times 10$ hexagons and (pbc, fbc).

Note that for the (fbc, pbc) choice, there is one site per hexagon at the boundary with only two instead of the usual $\Delta=3$ bonds. For the (pbc, fbc) BC's, there are two of these sites for each of the hexagons on the upper and lower boundaries. This motivated us to formulate a third kind of boundary condition: starting from the (pbc, fbc) choice, we added bonds connecting the boundary sites with fewer than three bounds so that all sites on the lattice have the same coordination number $\Delta=3$. We denote this choice as $(\mathrm{pbc}, \mathrm{fbc})_{\Delta}$. The zeros calculated with this third choice of boundary conditions are plotted in Fig. 3. The main difference relative to the previous two choices of BC's is that the zeros in the $\operatorname{Re}(a) \geq 0$ half plane have less scatter, lie closer to the exact boundary $\mathcal{B}$, and also, in some cases, lie inside the PM-FM phase boundary. The zeros with $\operatorname{Re}(a) \leq 0$ are less scattered than those with the (pbc,fbc) choice and track the arc of the unit circle well, although they do not, in general, lie on it, as was the case with the choice (fbc,pbc). The conclusion from this comparison with exactly known results is that, if one did not know the exact boundary $\mathcal{B}$ to begin with, one would be able, by combining results on zeros calculated with different boundary conditions, to reconstruct it with reasonable accuracy.

The density of zeros on $\mathcal{B}$ near the physical PM-FM transition is consistent with vanishing according to eq. (2.6) with $\alpha=0$, i.e., $g \sim\left|a-a_{P M-F M, q=2}\right|$ as $\left|a-a_{P M-F M, q=2}\right| \rightarrow 0$. By the $a \rightarrow 1 / a$ symmetry, the same is true of the PM-AFM transition. The situation at $z=-1$ is more complicated because the partition function has an isolated zero of multiplicity scaling like the lattice size there; consequently, as discussed above, the density $g$ has a delta function 
term $\propto \delta(s)$ as well as its usual term (2.6), where $s$ denotes the arclength on $\mathcal{B}$ away from the point $z=-1$. The analysis of Ref. [31 found that at $z=-1$, aside from the leading singularity $\sim(1+z)^{-2}$ in the specific heat, there is also a subleading logarithmic divergence; it follows that the density of zeros on $\mathcal{B}$ near to $z=-1$ has, in addition to the delta function term, a term that vanishes like $s$. The zeros in Figs. 2H3 are consistent with this.

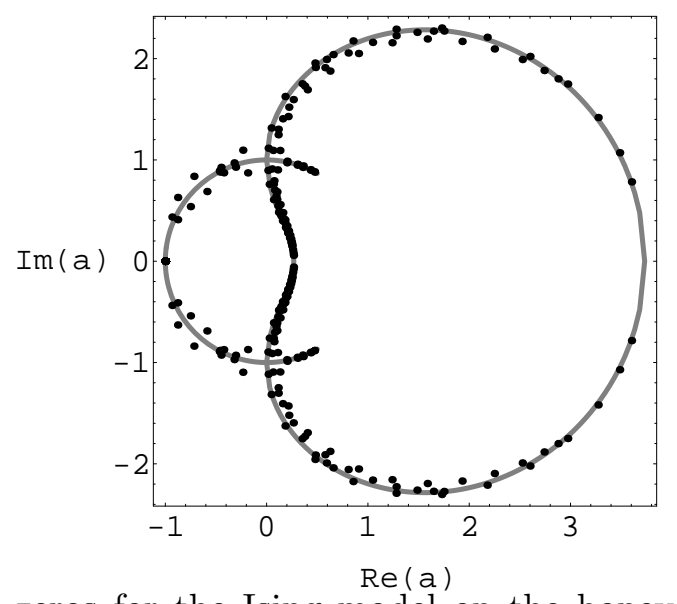

FIG. 3. Partition function zeros for the Ising model on the honeycomb lattice of size $10 \times 10$ hexagons and $(\mathrm{pbc}, \mathrm{fbc})_{\Delta} \mathrm{BC}$ 's.

\section{B. $q=3$ Case}

For general $q$, from duality and a star-triangle relation, an equation yielding the value of the PM-FM transition point has been derived [22], viz., $x^{3}-3 x-\sqrt{q}=0$, or, in terms of $a$,

$$
a^{3}-3 a^{2}-3(q-1) a-q^{2}+3 q-1=0
$$

For $0<q<4$, this equation has three real roots, while for $q>4$ (and the formal values $q \leq 0$ ) it has one real root. The motion of the real roots as a function of $q$ is plotted in Fig. 4. 


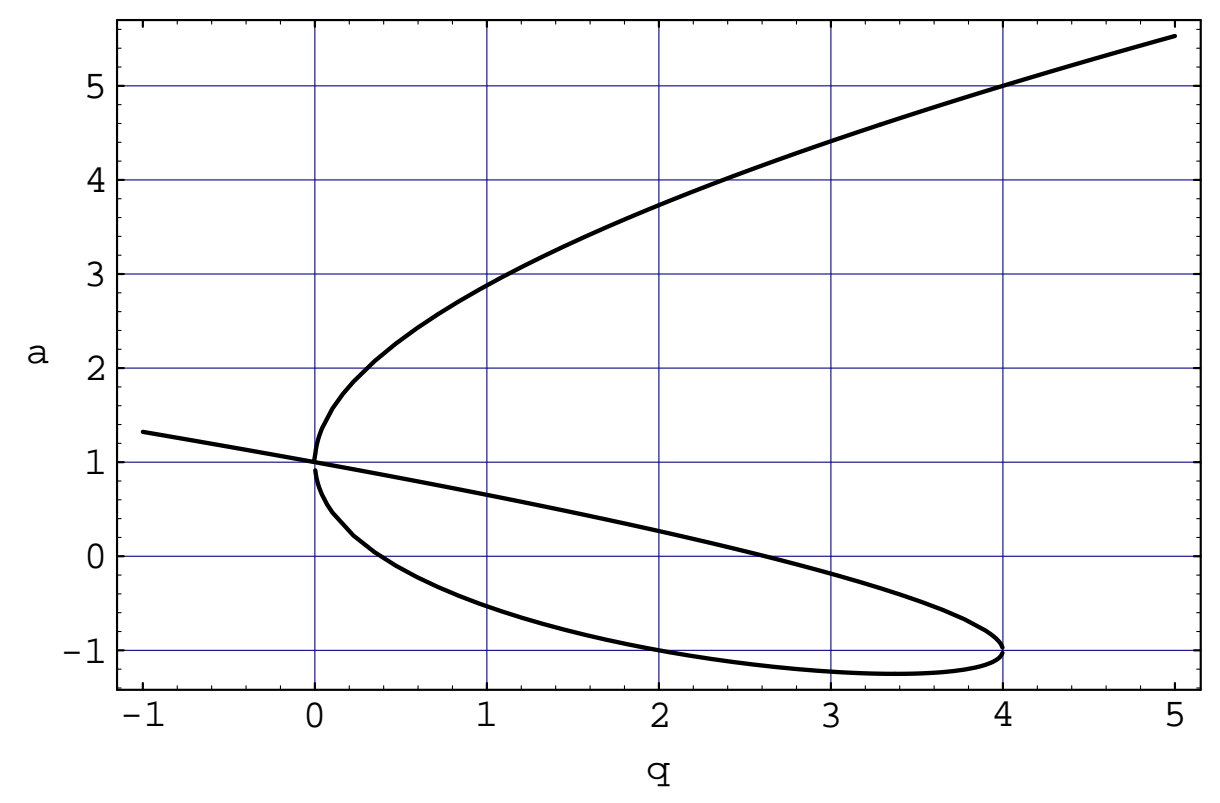

FIG. 4. Real roots of eq. (3.2), as a function of $q$.

For $q=3$, the solutions are

$$
\begin{gathered}
a_{1, q=3}=a_{P M-F M, q=3}=1+2 \sqrt{3} \cos (\pi / 18)=4.41147 \ldots \\
a_{2, q=3}=1-\sqrt{3} \cos (\pi / 18)+3 \sin (\pi / 18)=-0.1847925 \ldots
\end{gathered}
$$

and

$$
a_{3, q=3}=1-\sqrt{3} \cos (\pi / 18)-3 \sin (\pi / 18)=-1.22668 \ldots
$$

The point $a_{P M-F M, q=3}$ is the physical PM-FM critical point. As discussed in Ref. [29], if one follows the roots of eq. (3.2) as $q$ is changed continuously, one sees that the middle root $a_{2}$ decreases from the PM-AFM critical point $2-\sqrt{3}$ for $q=2$ through 0 at $q_{z}=(3+\sqrt{5}) / 2=$ $2.618 \ldots$ to the negative value (3.4) for $q=3$. This reflects the fact that as $q$ increases from 2 to $q_{z}$, the physical AFM phase is squeezed out.

Our zeros of the partition function for the $q=3$ case on the honeycomb lattice are shown

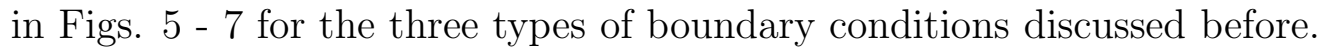




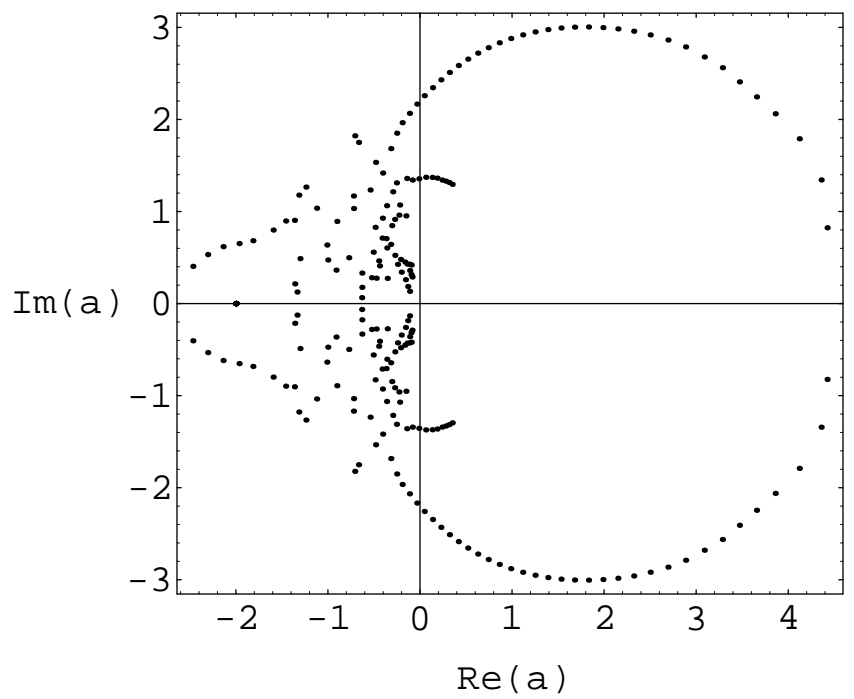

FIG. 5. Partition function zeros in the $a$ plane for the $q=3$ Potts model on a honeycomb lattice of size $8 \times 6$ hexagons and boundary conditions of type (fbc,pbc).

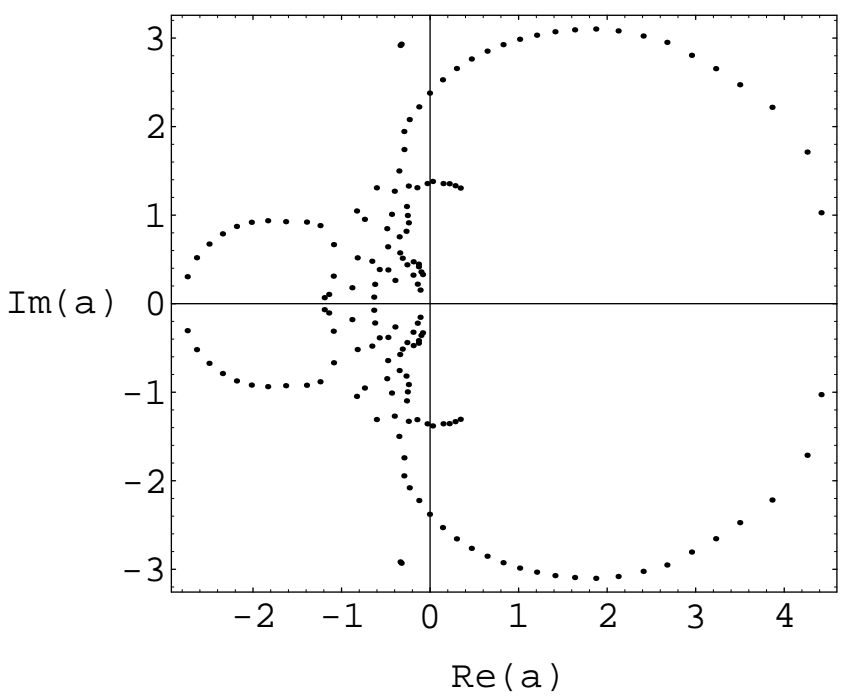

FIG. 6. Partition function zeros in the $a$ plane for the $q=3$ Potts model on a honeycomb lattice of size $8 \times 6$ hexagons and boundary conditions of type (pbc,fbc). 


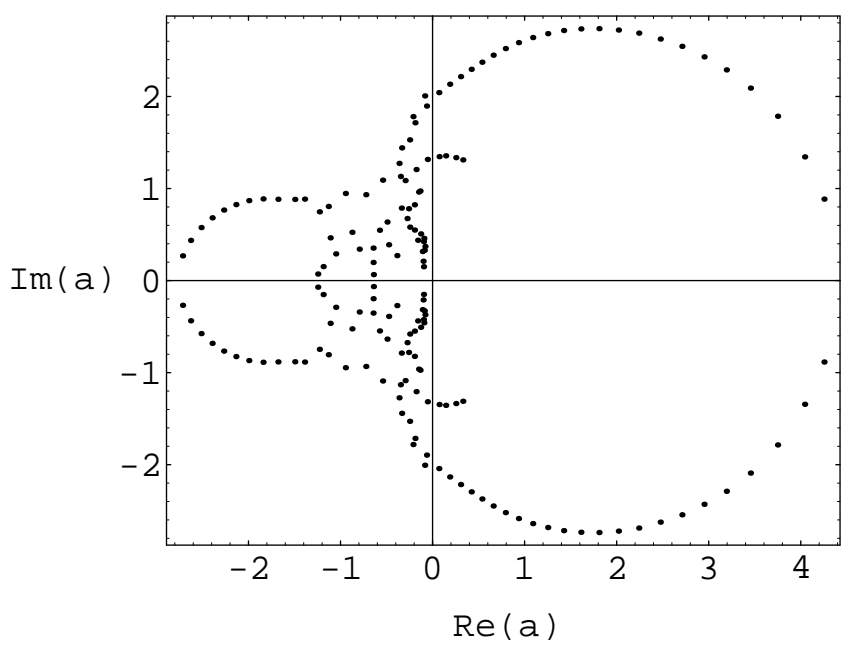

FIG. 7. Partition function zeros in the $a$ plane for the $q=3$ Potts model on a honeycomb lattice of size $8 \times 6$ hexagons and boundary conditions of type $(\mathrm{pbc}, \mathrm{fbc})_{\Delta}$.

In all three plots, the roots $a_{P M-F M, q=3}$ and $a_{3, q=3}$ correspond very well to points where the $\mathrm{CT}$ phase boundaries comprising $\mathcal{B}$ (as inferred for the thermodynamic limit from these zeros on finite lattices) cross the real axis. Hence, one anticipates that a CT phase boundary might cross the real axis at the value of the middle root, $a_{2, q=3}=-0.1848 \ldots$ From the zeros in Figs. 5 - 7, one can indeed infer that in the thermodynamic limit a CT phase boundary curve could cross the real axis at this point. Since the specific heat exponent has the known value $\alpha=1 / 3$ for this model [2], it follows from eq. (2.6) that the density of zeros near the physical PM-FM transition point vanishes like $g(s) \sim s^{2 / 3}$, where $s$ is the arclength on $\mathcal{B}$ away from this point. This is consistent with the calculated zeros.

Further, we see arcs protruding into the PM phase, ending at complex conjugate (c.c.) points $a_{e}, a_{e}^{*}=0.37(2) \pm 1.29(3) i$, where the numbers in parentheses refer to the estimated uncertainties in the final digits. Evidently, these are the analogues for $q=3$ of the exactly known $\operatorname{arcs}$ for the Ising $q=2$ case. While the arcs in the Ising case have endpoints on the unit circle at angles $\theta= \pm \pi / 3$, the endpoints in the $q=3$ case lie slightly farther out from the origin, at $\left|a_{e}\right| \simeq 1.3$, and have slightly larger angles $\theta \sim \pm 75^{\circ}$. We find that this trend is true for larger $q$ values also, i.e., $\left|a_{e}\right|$ and $\arg \left(a_{e}\right)$ increase with increasing $q$.

In addition, there are at least two more points at which curves of zeros cross the real $a$ axis, at $a=a_{\ell}=-2.77(3)$ and at $a=-0.65(2)$. In Ref. [13] it was shown that if the $q$-state Potts antiferromagnet on the dual lattice $\Lambda_{d}$ has a PM-AFM transition at $a_{P M-A F M, \Lambda_{d}}$, then the dual image of this, namely, $\mathcal{D}\left(a_{P M-A F M, \Lambda_{d}}\right)=a_{\ell, \Lambda}$, is the leftmost point in the $a$ plane where $\mathcal{B}$ crosses the real axis. Since the PM-AFM point satisfies $0 \leq a_{P M-A F M, \Lambda_{d}}<1$, it follows that the dual image $a_{\ell, \Lambda}$ satisfies $-\infty<a_{\ell, \Lambda} \leq-(q-1)$. In particular, for $q=3$, this 
connection was used, in conjunction with a precisely measured value of $a_{P M-A F M, t, q=3}$ on the triangular $(t)$ lattice [37] to infer the value of $a_{\ell}$ for the model on the honeycomb (hc) lattice: $a_{h c, \ell, q=3}=\mathcal{D}\left(a_{P M-A F M, t, q=3}\right)=-(2.76454 \pm 0.00015)$, i.e., $z_{h c, \ell, q=3}=a_{h c, \ell, q=3}^{-1}=-(0.36172 \pm$ 0.00002). Our zeros are in agreement with this result. This point also manifests itself as a singularity evident from low-temperature series for the specific heat $C$, magnetization $m$, and susceptibility $\chi$, which yield the value $z=-0.363 \pm 0.003$ [3]. Using duality and the weakly first order nature of the physical PM-AFM transition of the $q=3$ Potts antiferromagnet, it follows that the free energy also has the same weakly first order singularity at $a_{h c, \ell, q=3}$. The low-temperature series analysis of Ref. [3] found evidence for a continuous transition at this point, with exponents $\alpha_{\ell}=0.5, \beta_{\ell}=0.11$, and $\gamma_{\ell}=1.15$. We have repeated the series analysis with dlog Padé approximants (PA's) and differential approximants (DA's) [38]. Our DA results also yield $\alpha \simeq 0.5$; our PA's did not locate the singularity with sufficient precision to infer a reliable value for $\alpha$. Given the duality and the fact that $\alpha=\alpha^{\prime}$ for the physical PM-AFM transition of the $q=3$ Potts AF on the triangular lattice, it follows that the singularity in the free energy of the $q=3$ Potts model on the honeycomb lattice at $a_{\ell}$ must also be the same as approached from the right or left. Since the singularity in the internal energy at a singular point $a_{s}$ is $U_{\text {sing }} \sim\left|a-a_{s}\right|^{1-\alpha}$, one normally assigns the formal value $\alpha=1$ to a first-order transition. A possible way of reconciling these results is to observe that if a first order transition occurs superimposed with a divergent specific heat, then one could get a value of $\alpha<1$ in fitting the transition. For example, consider an illustrative internal energy function that behaves near a phase transition point like

$$
U(T) \sim U_{\text {analytic }}+c_{1,+} \Theta\left(T-T_{c}\right)+c_{2,+}\left|T-T_{c}\right|^{1 / 2}
$$

for $T \searrow T_{c}$, and similarly for $T \nearrow T_{c}$, with the coefficients replaced by $c_{1,-}$ and $c_{2,-}$. Here, $U_{\text {analytic }}$ denotes terms that are analytic near $T_{c}$ and $\Theta(x)$ is the step function, $\Theta(x)=1$ if $x>0$ and 0 otherwise. As one approached $T_{c}$ from above (below) a high-temperature (low-temperature) series analysis would give $\alpha=1 / 2$, but the transition would still be first order because of the discontinuous term. A one-sided version of this behavior occurs in the in the six-vertex model for the ferroelectric compound potassium dihydrogen phosphate (KDP) [39; in that case, the form (3.6) applies for the high-temperature side, while $U$ is a constant on the low-temperature side.

Another source of information on $\alpha$ is the density of zeros. However, it is difficult to use this to obtain an accurate value of $\alpha$. For example, Ref. [19] included calculations of zeros for the $q$-state square-lattice Potts model not just for the values $q=3,4$ where the PM-FM transition is continuous, but also for the values $q=5$ and 6 , where this PM-FM transition is first order; see Figs. 3 and 4 therein. For these cases, one would formally set 
$\alpha=1$ as mentioned above, so that eq. (2.6) would predict that the density $g$ of zeros should remain essentially constant up to the endpoint of the distribution (of course, the positivity of the coefficients of the terms in the partition function means that for a finite lattice, there cannot be any zeros on the positive real axis in the $a$ or $z$ plane). This is consistent with the plots of zeros for these $q=5$ and 6 cases, but it would be difficult to extract an accurate estimate of $\alpha$ from those plots. Below we shall present a similar plot for another case where the PM-FM transition is known to be first order, namely the $q=5$ Potts model on the honeycomb lattice, and a similar comment applies to this plot.

Further CT singularities and their relation with the boundary $\mathcal{B}$ will be discussed elsewhere in work with the authors of Ref. [3]. Our calculations also suggest that there are several unphysical $\mathrm{O}$ phases which overlap with parts of the negative real $a$ axis. There may be other $\mathrm{O}$ phases that do not touch the real axis, but the resolution is not high enough to make a definitive statement here.

Concerning the sensitivity of the zeros to lattice boundary conditions, several remarks are in order. The zeros in the $\operatorname{Re}(a) \geq 0$ half plane are relatively insensitive to these boundary conditions. However, certain features of the zeros in the $\operatorname{Re}(a)<0$ half plane do show such sensitivity. This is similar to what was found from a comparative study of different boundary conditions for the zeros of $Z$ for the $q$-state Potts model on the square lattice for several values of $q$ [17, 19] (see also Ref. [40]).

We have carried out similar calculations of zeros for the $q=4$ Potts model on the honeycomb lattice, and these will be reported in joint work with the authors of Ref. [3]. 


\section{C. $q=5$ Case}

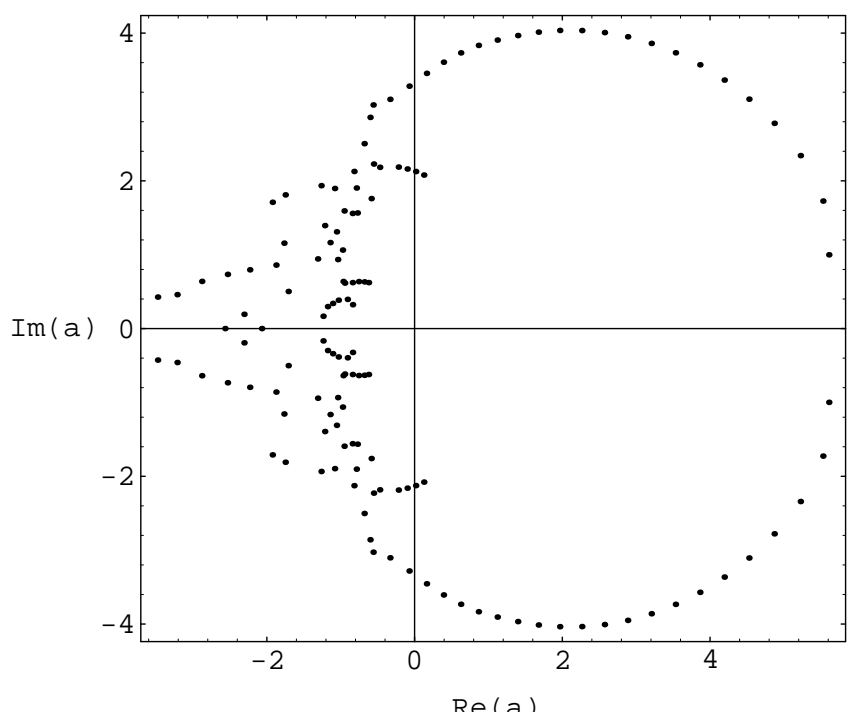

FIG. 8. Zeros of $Z$ in the $a$ plane for the $q=5$ Potts model on a honeycomb lattice of size $7 \times 6$ hexagons and boundary conditions of type (fbc,pbc).

It is also of interest to investigate a value of $q$ in the range where the PM-FM transition is first order, i.e., $q \geq 5$. We have done this for the value $q=5$, and we show a resulting plot of zeros in Fig. 8. Here, eq. (3.2) has the single real root, which is the PM-FM critical point,

$$
a_{P M-F M, q=5}=2^{-1 / 3} \cdot 5^{1 / 2}\left(1+5^{1 / 2}\right)^{1 / 3}+2^{1 / 3} \cdot 5^{1 / 2}\left(1+5^{1 / 2}\right)^{-1 / 3}+1=5.5298 \ldots
$$

Since the Potts antiferromagnet with $q=3$ and $q=4$ on the triangular lattice has, respectively, a finite-temperature PM-AFM phase transition [41,37] and a zero-temperature critical point [25], it is expected that for $q \geq 5$, the model is disordered for all temperatures. This, together with the connection discussed in Ref. [13], would imply that the leftmost point at which $\mathcal{B}$ crosses the real axis for the $q=5$ Potts model on the honeycomb lattice is $a_{\ell}<-4$. Our zeros are consistent with this.

\section{Further Discussion}

It is a general feature of the maximal (or sole) real solution of eq. (3.2), i.e., $a_{P M-F M}$, that it increases monotonically with $q$ for $q \geq 0$. This is evident in Fig. 4 and reflects the basic thermodynamic property that as $q$ increases, the spins become "floppier", and one 
must go to lower temperature to obtain FM long range order. In addition to the features already discussed, we note that (i) the leftmost point where $\mathcal{B}$ crosses the real $a$ axis, $a_{\ell}$, moves to the left as $q$ increases; and (ii) the points where $\mathcal{B}$ crosses the imaginary axis move out from the origin as $q$ increases. Both of these features can be understood, as discussed directly above, by the reduction in the size of the (FM) ordered phase as $q$ increases.

Because of the duality relation, these partition function zeros, in the $a$ plane, of the $q$ state Potts model on the honeycomb lattice also yield equivalent zeros of the same model on the dual, triangular lattice in the plane of the variable $a_{d}$ given in eq. (2.5). A comparison of the plots calculated with different boundary conditions is valuable since this gives a measure of the effects of these boundary conditions (see also Refs. [16,40]).

\section{PARTITION FUNCTION ZEROS ON THE KAGOMÉ LATTICE}

\section{A. Comparison with Exact $\mathcal{B}$ for Ising $q=2$ Case}

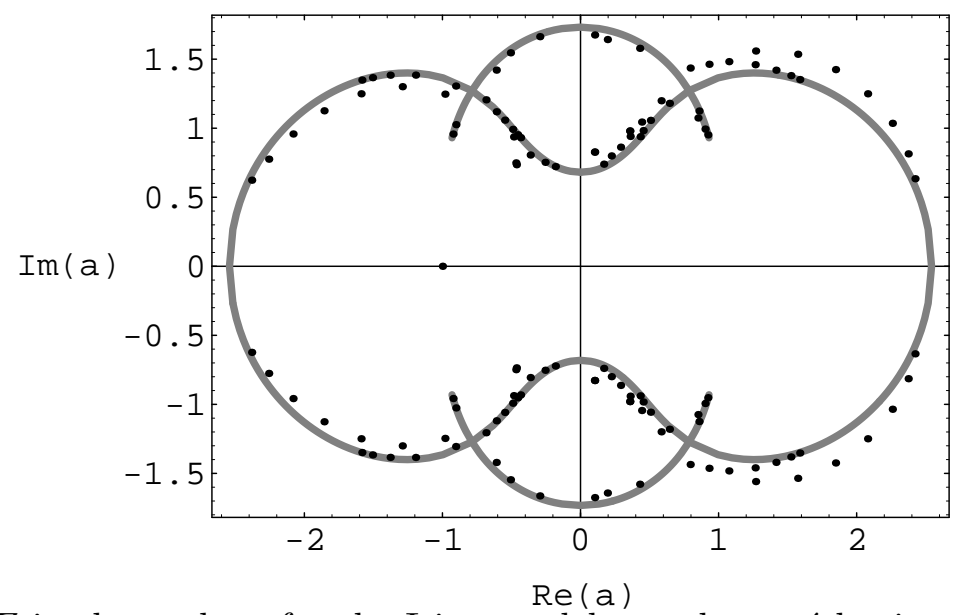

FIG. 9. Zeros of $Z$ in the $a$ plane for the Ising model on a kagomé lattice with $4 \times 6$ hexagons and (pbc,fbc) boundary conditions. 


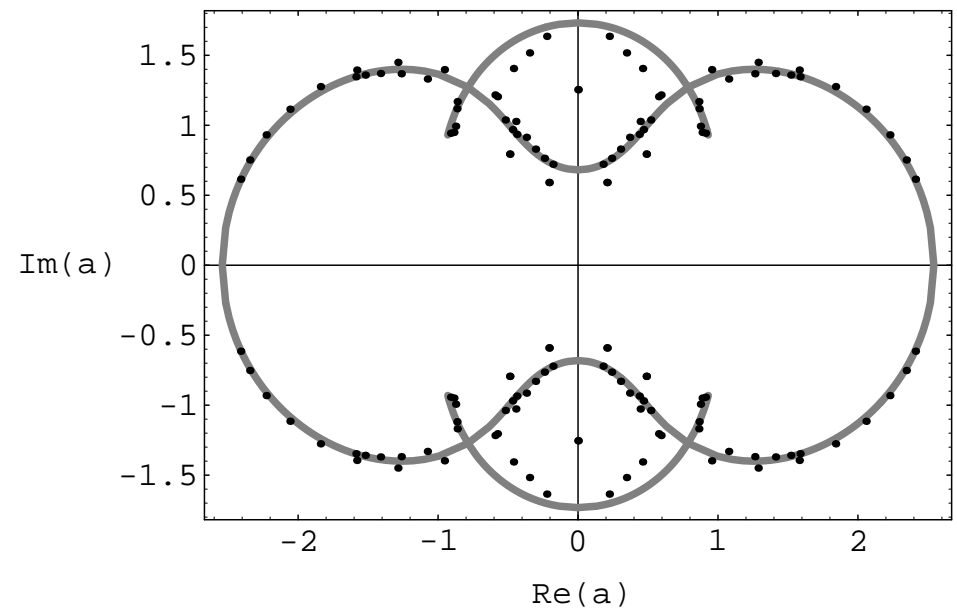

FIG. 10. Zeros of $Z$ in the $a$ plane for the Ising model on a kagomé lattice with $4 \times 6$ hexagons and $(\mathrm{pbc}, \mathrm{fbc})_{\Delta}$ boundary conditions.

For our calculations of zeros of Potts model on the kagomé lattice, two of the boundary conditions that we use are $(\mathrm{pbc}, \mathrm{fbc})$ and $(\mathrm{pbc}, \mathrm{fbc})_{\Delta}$, where now $\Delta=4$. The third can be described as follows: we start on the lattice that is the dual to kagomé, namely the diced lattice, and impose (pbc,fbc) boundary conditions; then we transform the results back to the kagomé lattice by the duality map on $a$, eq. (2.5). To save space, for each value of $q$, we only show results for the first two of these choices of boundary conditions. For $q=2$ these are given in Figs. 9 and 10 . The exact CT phase boundary $\mathcal{B}$ is given by the locus of solutions of the equation

$$
a^{8}+18 a^{4}+24 a^{2}+21-4\left(1+a^{2}\right)\left(1-a^{2}\right)^{2} p=0
$$

where $-3 / 2 \leq p \leq 3$ [32]. Because the coordination number of the kagomé lattice is even, this locus is symmetric under $a \rightarrow-a$. In Ref. [11], the locus was plotted in the $z$ and $u=z^{2}$ planes (see also Ref. 442). Here it is shown as the gray curves in the $a$ plane, consisting of a "dumbell" part and a complex conjugate pair of circular arcs which intersect the dumbell at four multiple points (the analytic expressions for which are given in Ref. [11]). The inside of the dumbell region is the PM phase, the c. c. regions between the narrow neck of the dumbell and the circular arcs are $\mathrm{O}$ phases, and the region outside of $\mathcal{B}$ and extending to complex infinity is the (CT extension of the) FM phase. The PM-FM critical point is given by $a_{k a g, P M-F M, q=2}=-a_{k a g, \ell, q=2}=3^{1 / 4}(2-\sqrt{3})^{-1 / 2}=2.542 \ldots$ Just there is no physical AFM phase (owing to the frustration of the Ising AF on the kagomé lattice), so also there is no complex-temperature extension thereof. Comparing the zeros calculated with the different boundary conditions, we find that with the (pbc,fbc) choice, the zeros on the neck of the dumbell and on the outer circular arcs track the exact curves well, while those on the right 
(left) lie slightly outside (inside) the CT phase boundaries. With the (pbc,fbc) boundary conditions, not all sites have even coordination number, so that $Z$ contains some odd powers of $a$, and hence the $a \rightarrow-a$ symmetry of the exact boundary is not precisely maintained by the zeros. In passing, we note that because the sites on the upper and lower boundaries have odd coordination number $\Delta=3$, theorem 6 of Ref. [11 implies that $Z(z=-1)=0$, and this zero (which is multiple) is evident in Fig. 9. For the (pbc,fbc) $)_{\Delta}$ boundary conditions, (i) most of the zeros near to the dumbell lie closer to the exact curves, but the zeros near the arcs lie farther away from them, as compared with the situation for the (pbc,fbc) choice; and (ii) $Z$, and hence its zeros, is invariant under the negation $a \rightarrow-a$, in contrast to case with the (pbc,fbc) case; (iii) because all sites have even coordination number, there is no zero in $Z$ at $z=-1$. For both types of boundary conditions, the density of zeros in the vicinity of the PM-FM critical point $a_{k a g, P M-F M, q=2}$ decreases in a manner consistent with the form from eq. (2.6) with $\alpha=0$ for the 2D Ising model, viz., $g \sim s$ as $s \rightarrow 0$, where $s$ the arclength along $\mathcal{B}$ away from $a_{P M-F M, q=2}$.

\section{B. $q=3$ Case}

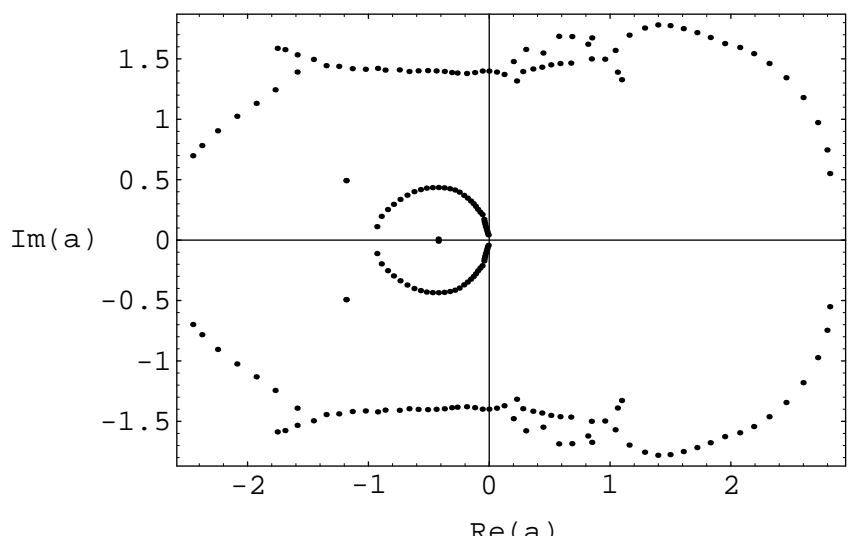

FIG. 11. Zeros of $Z$ in the $a$ plane for the $q=3$ Potts model on a kagomé lattice of size $4 \times 8$ hexagons and (pbc,fbc) boundary conditions. 


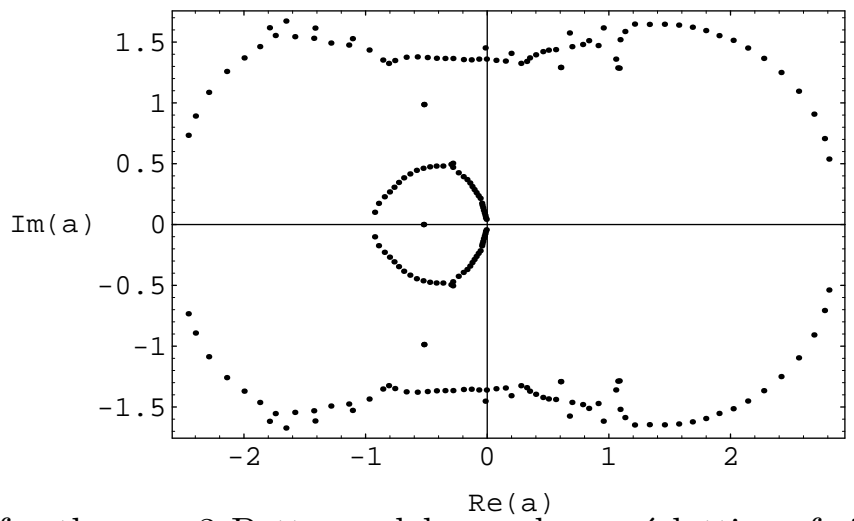

FIG. 12. Zeros of $Z$ for the $q=3$ Potts model on a kagomé lattice of size $4 \times 8$ hexagons and $(\mathrm{pbc}, \mathrm{fbc})_{\Delta}$ boundary conditions.

We show our zeros of $Z$ for the $q=3$ Potts model on the kagomé lattice in Figs. 11 and 12. In this case we use lattices of sizes $N_{x} \times N_{y}$ with $N_{y}$ larger than $N_{x}$ in order to compensate for the fact that the free boundaries are in the $y$ direction and free, as contrasted with periodic, boundary conditions introduce greater finite-size effects. The zeros suggest that in the thermodynamic limit, the inferred CT phase diagram for the $q=3$ kagomé lattice may involve somewhat simpler boundary curves than was the case for the same model on the honeycomb lattice. There is a high-temperature PM phase, a low-temperature FM phase, and there are strong indications of a third CT phase whose right-hand boundary crosses the real axis at $a=0$, corresponding to a zero-temperature critical point of the $q=3$ Potts antiferromagnet on this lattice. This is in good agreement with the known property that this model does have such a $T=0$ critical point (which can be related to the $T=0$ critical point of the $q=4$ Potts antiferromagnet on the triangular lattice) [13,44]. The inferred position where the CT boundary crosses the real axis on the right is at $a_{k a g, P M-F M, q=3}=2.84(4)$. This is in accord, to within the uncertainty, with the value of $a_{k a g, P M-F M, q=3}=2.87646(4)$, i.e., $\left.z_{\text {kag }, P M-F M, q=3}=0.347650(5)\right)$ obtained from series analysis [3]. The left-hand boundary of the third phase crosses the real axis at about $a=-0.96(3)$. From Fig. 12, where the boundary between the (CT extensions of the) PM and the FM phases is probably best represented, we infer that the leftmost point where this CT phase boundary crosses the real axis is at $a_{k a g, \ell, q=3}=-2.54(6)$. This point is manifested as a singularity in thermodynamic quantities evident in low-temperature series analysis, which obtains $a_{k a g, \ell, q=3}=-2.486(3)$ (i.e., $\left.z_{k a g, \ell, q=3}=-0.4023(5)\right)$. Although the CT phase boundary is not symmetric under $a \rightarrow-a$ as was true for $q=2$ on this lattice, one can still discern a remnant of the dumbell shape that occurred for the $q=2$ case. As before for the honeycomb lattice with $q=3$, the $g \sim s^{2 / 3}$ decrease in the density of zeros in the vicinity of the PM-FM critical point is 
consistent with the calculated zeros.

\section{Case $q=4$}

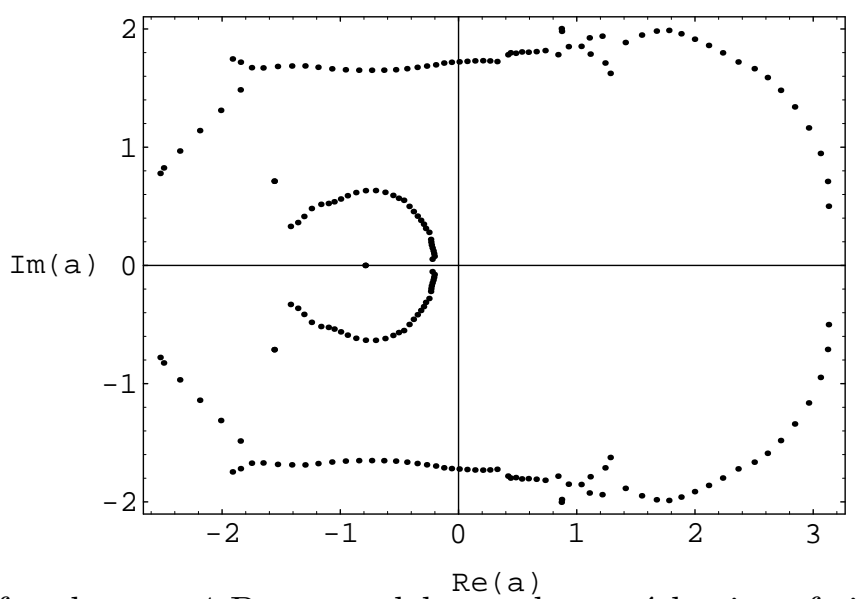

FIG. 13. Zeros of $Z$ for the $q=4$ Potts model on a kagomé lattice of size $4 \times 8$ hexagons and (pbc,fbc) boundary conditions.

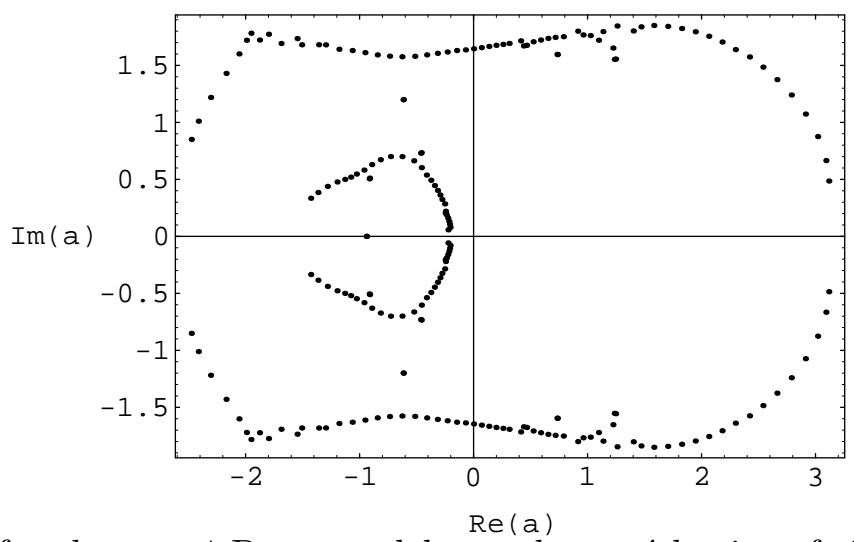

FIG. 14. Zeros of $Z$ for the $q=4$ Potts model on a kagomé lattice of size $4 \times 8$ hexagons and $(\mathrm{pbc}, \mathrm{fbc})_{\Delta}$ boundary conditions.

For $q=4$, we present our results in the Figs. 13 and 14. The main differences between the locus of zeros, as compared with the case of $q=3$ is, first, that the crossing which occurs at $a=0$ for $q=3$ is shifted to a negative value, $a=-0.21(2)$ for $q=4$. This demonstrates that the $q=4$ Potts antiferromagnet on the kagomé lattice has no PM-AFM phase transition (or any hypothetical non-symmetry breaking phase transition) at finite temperature or any critical point at $T=0$. This conclusion also follows for $q=5$ since increasing $q$ beyond 4 
has the effect of making the spins "floppier" and the model more disordered. For $q \geq 6$, this conclusion has been proved rigorously [28]. Second, it appears that the previously presumably closed inner ring of zeros has now opened at its leftmost point, which would imply that now there would be only two phases (with their CT extensions), the PM and FM. The values inferred for $a_{k a g, P M-F M, q=4}$ and $a_{k a g, \ell, q=4}$ are in accord with the values obtained from series analysis [3]. Since the specific heat critical exponent $\alpha=2 / 3$ for the

$q=4$ Potts model on 2D lattices, eq. (2.6) gives $g \sim s^{1 / 3}$ for the manner in which the density of zeros vanishes as one approaches the PM-FM critical point along the CT phase boundary. In particular, this implies that the decrease in density should be less rapid for $q=4$ than for $q=3$, and, indeed, this is evident from a comparison of our plots of zeros for these two cases on the kagomé lattice.

\section{Partition Function Zeros on the Diced Lattice}

As with the honeycomb lattice and its dual, the triangular lattice, our zeros, in the $a$ plane, of the partition function for the $q$-state Potts model on the kagomé lattice also yield equivalent zeros of the same model on the lattice that is dual to the kagomé lattice, in the plane of the variable $a_{d}$ given in eq. (2.5). Henceforth, we shall suppress the subscript $d$ on $a_{d}$. This dual lattice is called the diced lattice; as discussed above, it is not an Archimedean lattice [36]; rather, it is a tiling of the plane with identical rhombi such that, as one traverses a circuit along the edges of each rhombus, one passes vertices with coordination number $3,6,3,6$ in sequence. Thus, in standard mathematical notation, the diced lattice is the lattice $[3 \cdot 6 \cdot 3 \cdot 6]$ dual to the $(3 \cdot 6 \cdot 3 \cdot 6)$ (= kagomé) lattice. Some relevant properties of the diced lattice are noted in (Table II of) Ref. [27]. Although the faces of the diced lattice are identical, the vertices are not (this is the dual of the property that the vertices of an Archimedean lattice are identical but the faces are, in general, not, since an Archimedean lattice can consist of more than one type of regular polygon). In particular, the diced lattice has vertices of two different types: one with an odd degree (= coordination number) $\Delta=3$, and the other with even degree, $\Delta=6$. Indeed, the diced lattice is bipartite, and its two sublattices, which we may denote $\Lambda_{3}$ and $\Lambda_{6}$, are comprised of the vertices with degree $\Delta=3$ and $\Delta=6$, respectively. The vertices in the $\Lambda_{3}$ and $\Lambda_{6}$ sublattices occupy the respective fractions $f_{3}=2 / 3$ and $f_{6}=1 / 3$ of all the vertices. This is quite different from bipartite Archimedean lattices, where the vertices on each of the two sublattices occupy the same fraction, $f=1 / 2$, of the total number of vertices (as a consequence of the fact that on an Archimedean lattice, all vertices are equivalent). 


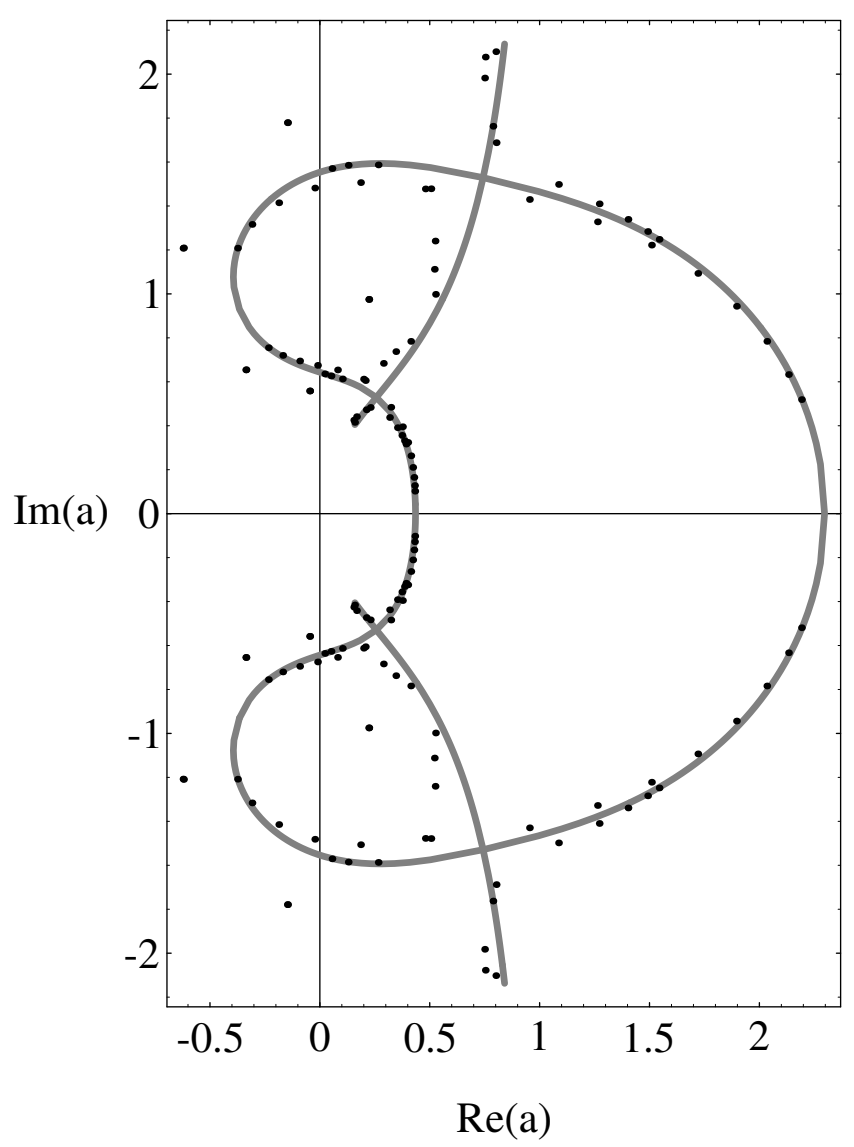

FIG. 15. Zeros of $Z$ for the Ising model on a diced lattice, obtained via duality from a kagomé lattice of size $4 \times 8$ hexagons and $(\mathrm{pbc}, \mathrm{fbc})_{\Delta}$ boundary conditions.

For our discussion of the CT phase diagrams of the $q$-state Potts model with $q=3,4$ on the diced lattice, it is instructive to begin by discussing the $q=2$ case, for which one can use exact results on the free energy. The CT phase diagram is shown in Fig. 15. The exact CT phase boundary $\mathcal{B}$ [42, 11$]$ is shown as the dark solid curve. Using duality and the $z \rightarrow-z$ symmetry of the boundary for the Ising model on the kagomé lattice (the latter of which follows from the even coordination number of that lattice), it follows that the $\mathcal{B}$ in Fig. 15 for the Ising model on the diced lattice is the same as $\mathcal{B}$ in the $v$ plane for the model on the kagomé lattice, where $v=(1-z) /(1+z)$. Note that although the physical FM and AFM phases are disjoint, the respective complex-temperature extensions of these phases are analytically connected. The reason for this is that, in contrast to bipartite Archimedean lattices, the two sublattices $\Lambda_{3}$ and $\Lambda_{6}$ of the diced lattice do not occupy the same fraction of the total lattice. Thus, reverting to conventional Ising model notation for this discussion, let us define $M_{\Lambda_{3}}$ and $M_{\Lambda_{6}}$ as the magnetizations of the sublattices $\Lambda_{3}$ and $\Lambda_{6}$ and $M_{u n i f}$. and $M_{\text {stag. }}$ as the uniform and staggered magnetizations, all per unit area of the total lattice, 
with

$$
\begin{aligned}
& M_{\text {unif. }}=M_{\Lambda_{3}}+M_{\Lambda_{6}} \\
& M_{\text {stag. }}=M_{\Lambda_{3}}-M_{\Lambda_{6}}
\end{aligned}
$$

If the present lattice had been Archimedean, with each sublattice occupying a fraction $1 / 2$ of the total, then $M_{\text {stag. }}$. would vanish identically not just in the PM phase but also in the FM phase, and $M_{\text {unif. }}$ would vanish identically not just in the PM phase but also the AFM phase, so that the FM and AFM phases, and their complex-temperature extensions, could not be analytically connected with each other. However, because the sublattices of the diced lattice occupy different fractions of the total lattice, it follows that in the limit of complete sublattice spin ordering, $M_{\Lambda_{3}}=2 / 3$ and $M_{\Lambda_{6}}=1 / 3$ and hence, besides the obvious result, $M_{\text {unif. }}(a=\infty)=1$, one has

$$
\begin{gathered}
M_{\text {unif. }}(a=0)=\frac{1}{3} \\
M_{\text {stag. }}(a=\infty)=\frac{1}{3} \\
M_{\text {stag. }}(a=0)=1
\end{gathered}
$$

That is, the uniform magnetization $M_{\text {unif. }}$ does not vanish even in the region of complete sublattice magnetizations of opposite sign, at $T=0$ for $J<0$, i.e., $a=0$, and the staggered magnetization $M_{\text {stag. }}$ does not vanish even in the limit of complete sublattice magnetizations of the same sign, at $T=0$ for $J>0$, i.e., $a=\infty$. Hence, there exist paths that connect the points $a=\infty$ and $a=0$ in the complex $a$ plane. Of course, if one restricts to the physical temperature interval $0 \leq a \leq \infty$, then the physical FM and AFM phases cannot be analytically connected, since they are separated by the PM phase, where both $M_{\text {unif. }}$ and $M_{\text {stag. }}$ vanish identically. However, the complex-temperature extensions of the FM and AFM phases are analytically connected, as is shown by the existence of the paths alluded to above.

The CT phase diagram is thus as follows: (see Fig. 15: first, there is a symmetric, hightemperature PM phase around the point $a=1$ that includes the interval $a_{1}<a<a_{1}^{-1}$ on the real axis, where

$$
a_{1}=\frac{1}{2}(1+\sqrt{3})\left[1-(2 \sqrt{3}-3)^{1 / 2}\right]=0.43542 \ldots
$$


Secondly, there is the single complex-temperature extension of the two different physical FM and AFM phases; this extension includes the intervals $-\infty \leq a<a_{1}$ and $a_{1}^{-1}<a \leq \infty$ on the real axis (see eq. (4.12) in Ref. [11]) and extends outward to complex infinity in the a plane. We label this phase as CT(A)FM. Third, there is a complex-conjugate pair of $\mathrm{O}$ phases. In Fig. 15 we have shown the zeros computed with one particular set of boundary conditions; in this case and also with the other types of boundary conditions, these zeros agree well with the exact results. (This is dual to the same statement for the $q=2$ kagomé lattice.)

Proceeding to the cases that have not been exactly solved, in Fig. 16 we show our zeros for the $q=3$ Potts model on the diced lattice, obtained via duality from those on the kagomé lattice. For this and $q=4$, we show results with only one set of boundary conditions, since the other boundary conditions yield similar results. As noted in Ref. [13], from the finding in Ref. [3] of a CT singularity at $z_{\ell}=-0.4023(5)$ in the $q=3$ Potts model on the kagomé lattice, it follows, using the duality connection, that the $q=3$ Potts model has a phase transition from the PM phase to the FM-AFM phase at the point $a_{\text {diced, } q=3, P M-A F M}=$ 0.1393(8). This constitutes the left border of the physical PM phase on the positive real $a$ axis. Moreover, again by duality, from the PM-FM transition point of the model on the kagomé lattice, determined from series analysis in Ref. [3] to be at $z_{c}=0.347650(5)$, it follows that the position of the PM-FM transition of the $q=3$ Potts model on the diced lattice is at $a_{\text {diced,PM } M-F M, q=3}=2.59876(4)$. As in the $q=2$ case, and for the same reason, although the physical FM and AFM phases are disjunct, their complex-temperature extensions are analytically connected. We thus again label this extension as the CT(A)FM phase. The other CT phases include the extension of the PM phase and an $\mathrm{O}$ phase in the $\operatorname{Re}(a)<0$ halfplane. Our finding that the CT phase boundary for the $q=3$ Potts model on the kagomé lattice has a component that passes through $a=0$, corresponding to a zero-temperature critical point in that model, implies, by duality, that the boundary of the $\mathrm{O}$ phase in the model on the diced lattice crosses the real $a$ axis on the left at $a=-2$.

We show our zeros for the $q=4$ Potts model on the diced lattice in Fig. 17. For this case, from the value $a_{k a g, P M-F M, q=4}=3.1561(5)$ obtained from series analysis in Ref. [3], we deduce, using duality, that the PM-FM critical point for the diced lattice is $a_{\text {diced, } P M-F M, q=4}=2.8552(5)$. Further, from the value obtained for the CT singularity, $z_{\text {kag }, q=4, \ell}=-0.42 \pm 0.01$ [3], we have deduced, again using duality, that the $q=4$ Potts antiferromagnet on the diced lattice has no finite- $T$ phase transition and also is not critical at $T=0$, since

$$
\mathcal{D}\left(a_{\text {kag }, q=4, \ell}\right)=-(0.18 \pm 0.02)
$$


is negative [13]. There is thus no AFM phase for $q=4$. Because increasing the value of $q$ for a fixed temperature makes the spins floppier, this result implies that there is also no AFM phase for $q \geq 5$. In the context of the complex-temperature phase diagram, the point in eq. (4.8) corresponds to the point where the two arcs close in the thermodynamic limit and the left-hand boundary of the PM phase crosses the real $a$ axis in Fig. 17. In this figure one also sees a curve in $\mathcal{B}$ in the $\operatorname{Re}(a)<0$ half-plane.

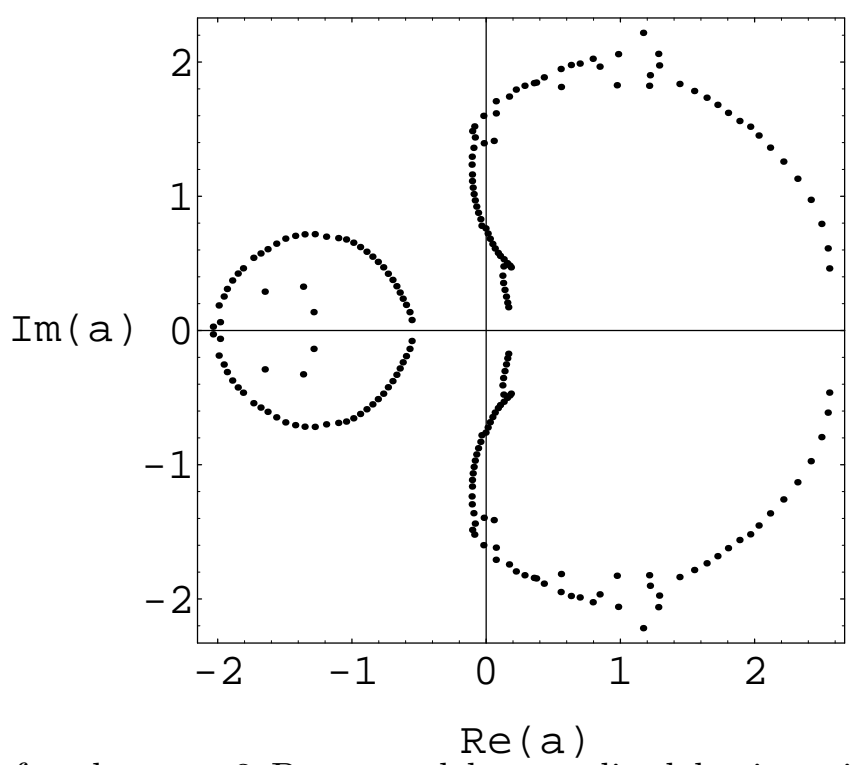

FIG. 16. Zeros of $Z$ for the $q=3$ Potts model on a diced lattice with (pbc,fbc) boundary conditions and of size equivalent to $4 \times 8$ hexagons on a kagomé lattice.

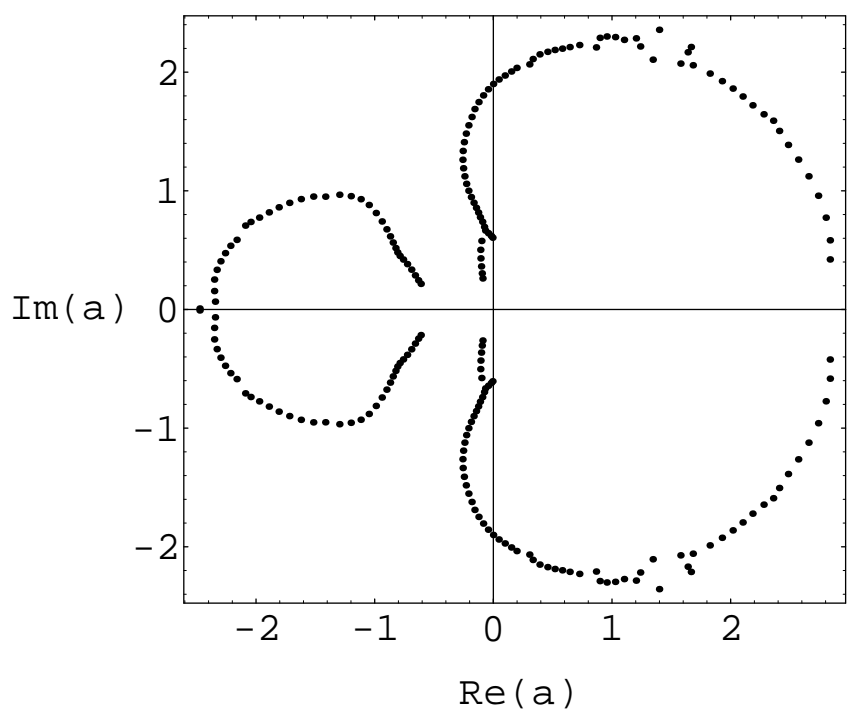


FIG. 17. Zeros of $Z$ for the $q=4$ Potts model on a diced lattice with (pbc,fbc) boundary conditions and of size equivalent to $4 \times 8$ hexagons on a kagomé lattice.

\section{CONCLUSIONS}

We have calculated complex-temperature zeros of the partition function for the $q$-state Potts model on the honeycomb and kagomé lattices. These results give useful information about the complex-temperature phase diagrams and singularities of these models.

\section{Acknowledgments}

This research was supported in part by the NSF grant PHY-97-9722101. R.S. thanks. Prof. A. J. Guttmann for kindly giving us a copy of Ref. [3] prior to publication and for discussions of that work.

[1] R. B. Potts, Proc. Camb. Phil. Soc. 48, 106 (1952).

[2] F. Y. Wu, Rev. Mod. Phys. 54, 235 (1982).

[3] I. Jensen, A. J. Guttmann, and I. G. Enting, "The Potts Model on Kagomé and Honeycomb Lattices", Melbourne preprint (1997). These authors use the symbol $u$ for what is denoted $z$ here.

[4] C. N. Yang and T. D. Lee, Phys. Rev. 87, 404 (1952); T. D. Lee and C. N. Yang, ibid 87, 410 (1952).

[5] M. E. Fisher, Lectures in Theoretical Physics (Univ. of Colorado Press, 1965), vol. 7C, p. 1.

[6] S. Katsura, Prog. Theor. Phys. 38, 1415 (1967); S. Ono, Y. Karaki, M. Suzuki, and C. Kawabata, J. Phys. Soc. Jpn. 25, 54 (1968).

[7] R. Abe, Prog. Theor. Phys. 38, 322 (1967). 
[8] C. Domb and A. J. Guttmann, J. Phys. C 3, 1652 (1970).

[9] V. Matveev and R. Shrock, J. Phys. A 28, 4859 (1995); Phys. Rev. E53, 254 (1996); Phys. Lett. A215 271 (1996).

[10] The free energy also has an isolated singularity at $|K|=\infty$ and (see Theorem 6 of Ref. [11]) for the Ising model on lattices with odd coordination number, a singularity at $z=-1$. The latter lies on the complex-temperature phase boundary for the honeycomb lattice but is isolated for the heteropolygonal $3 \cdot 12^{2}$ lattice.

[11] V. Matveev, and R. Shrock, J. Phys. A 28, 5235 (1995).

[12] As discussed in Ref. [14], the complex-temperature extension of a physical phase is obtained by analytically continuing the free energy from the interval of physical temperature to a maximal region allowed by nonanalytic boundaries. Henceforth, we shall generally take the adjective "complex-temperature extension" as implicit when referring to phases. There are also other complex-temperatures that have no overlap with any physical phase; we shall denote these by O for "other".

[13] H. Feldmann, R. Shrock, and S.-H. Tsai, J. Phys. A (Lett.) 30, L663 (1997).

[14] V. Matveev, and R. Shrock, J. Phys. A 28, 1557 (1995).

[15] G. Marchesini and R. Shrock, Nucl. Phys. B318, 541 (1989).

[16] P. P. Martin, and J.-M. Maillard, J. Phys. A 19 L547 (1986).

[17] P. P. Martin, Potts Models and Related Problems in Statistical Mechanics (World Scientific, Singapore) (1991).

[18] C. N. Chen, C. K. Hu, and F. Y. Wu, Phys. Rev. Lett. 76169 (1996); F. Y. Wu, G. Rollet, H. Y. Huang, J. M. Maillard, C. K. Hu, and C. N. Chen, Phys. Rev. Lett. 76, 173 (1996). 
[19] V. Matveev, and R. Shrock, Phys. Rev. E54, 6174 (1996).

[20] C. Itzykson, H. Saleur, and J.-B. Zuber, eds., Conformal Invariance and Applications to Statistical Mechanics (World Scientific, Singapore, 1988).

[21] T. Kihara, Y. Midzuno and T. Shizume, J. Phys. Soc. Japan 9, 681 (1954).

[22] D. Kim and R. J. Joseph, J. Phys. C 7, L167 (1974); T. W. Burkhardt and B. W. Southern, J. Phys. A 11 L247 (1978).

[23] R. J. Baxter, J. Phys. C 6, L445 (1973); R. J. Baxter, H. N. V. Temperley and S. Ashley, Proc. Roy. Soc. London, Ser. A 358, 535 (1978); R. J. Baxter, J. Stat. Phys. 28, 1 (1982).

[24] E. H. Lieb, Phys. Rev. 162, 162 (1967).

[25] R. J. Baxter, J. Phys. A 20, 5241 (1987); J. Phys. A 19, 2821 (1986).

[26] R. Shrock and S.-H. Tsai, Phys. Rev. E55, 5165, 6791 (1997); Phys. Rev. E56, 2733, 3935 (1997).

[27] R. Shrock and S.-H. Tsai, Phys. Rev. E56, 4111 (1997).

[28] J. Salas and A. Sokal, J. Stat. Phys. 86, 551 (1997).

[29] R. Shrock and S.-H. Tsai, J. Phys. A 30, 495 (1997).

[30] For the physical critical point, one does not have to distinguish between the exponents $\alpha$ and $\alpha^{\prime}$ describing the approach from the high-temperature and low-temperature sides, since these are equal.

[31] V. Matveev and R. Shrock, J. Phys. A 29, 803 (1996).

[32] In both the honeycomb and kagomé cases, the respective conditions (3.1) and (4.1) are the conditions that $L(a, p)=0$, where $L$ occurs in the term $\propto \int_{0}^{2 \pi} d \theta_{1} \int_{0}^{2 \pi} d \theta_{2} \ln [L(a, p)]$ in the free 
energy, and $p=\cos \left(\theta_{1}\right)+\cos \left(\theta_{2}\right)+\cos \left(\theta_{1}+\theta_{2}\right)$. The range of $p$ is then $-3 / 2 \leq p \leq 3$, as given.

[33] By duality, for the Ising model on the honeycomb lattice, $\mathcal{B}$ in $a$ plane is the same as $\mathcal{B}$ in the $a_{d}=(a+1) /(a-1)$ plane for same model on the triangular lattice [31]. Note that for the case of anisotropic spin-spin couplings, the partition function zeros fill out regions rather than lying on curves in the $a$ plane [34].

[34] J. Stephenson and R. Couzens, Physica 129A, 201 (1984); W. van Saarloos and D. Kurtze, J. Phys. A 17, 1301 (1984); J. Stephenson and J. van Aalst, Physica 136A, 160 (1986); J. Stephenson, Physica 148A, 107 (1988) and references therein.

[35] S. Lefschetz, Algebraic Geometry (Princeton Univ. Press, Princeton, 1953); R. Hartshorne, Algebraic Geometry (Springer, New York, 1977).

[36] B. Grünbaum and G. Shephard, Tilings and Patterns (Freeman, New York, 1987).

[37] J. Adler, A. Brandt, W. Janke, and S. Shmulyian, J. Phys. A 28, 5117 (1995).

[38] A. J. Guttmann, in C. Domb and J. Lebowitz, eds., Phase Transitions and Critical Phenomena (New York, Academic Press, 1989), vol. 13.

[39] B. Sutherland, Phys. Rev. Lett. 19, 103 (1967); E. H. Lieb, ibid., 19, 108 (1967); see also Fig. 17 in E. H. Lieb and F. Y. Wu, in C. Domb and M. S. Green, Phase Transitions and Critical Phenomena (Academic Press, New York, 1972), p. 331.

[40] P. P. Martin, J. Phys. A 19, 3267 (1986); ibid., 20, L601 (1986).

[41] G. S. Grest, J. Phys. A 14, L217 (1981); Y. Saito, J. Phys. A 15, 1885 (1982); I. G. Enting, and F. Y. Wu, J. Stat. Phys. 28, 351 (1982).

[42] R. Abe, T. Dotera, and T. Ogawa, Prog. Theor. Phys. 85, 509 (1991).

[43] C. Broholm, G. Aeppli, G. P. Espinoza, and A. S. Cooper, J. Appl. Phys. 69, 4968 (1991); D. 
A. Huse and A. D. Rutenberg, Phys. Rev. B45, 7536 (1992).

[44] J. Kondev and C. Henley, Nucl. Phys. B464, 540 (1996). 